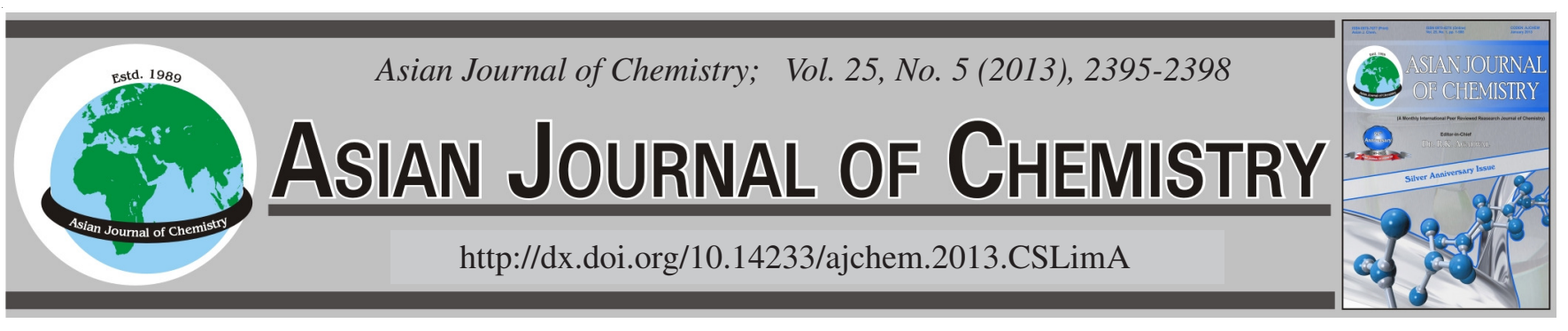

\title{
Microwave-Assisted Solvothermal Synthesis and Characterization of Barium Orthovanadate Nanoparticles
}

\author{
Chang Sung Lim
}

Department of Advanced Materials Science \& Engineering, Hanseo University, Seosan 356-706, South Korea

Corresponding author: Tel/Fax: +82 41 6601445; E-mail: cslim@ hanseo.ac.kr

\begin{abstract}
Barium orthovanadate $\left(\mathrm{Ba}_{3} \mathrm{~V}_{2} \mathrm{O}_{8}\right)$ nanoparticles were synthesized successfully using a facile microwave solvothermal route followed by further heat-treatment. Well-crystallized $\mathrm{Ba}_{3} \mathrm{~V}_{2} \mathrm{O}_{8}$ nanoparticles were formed after heat-treatment at $600{ }^{\circ} \mathrm{C}$ for $3 \mathrm{~h}$ showing a fine and homogeneous morphology with particle sizes of $50-150 \mathrm{~nm}$. The synthesized $\mathrm{Ba}_{3} \mathrm{~V}_{2} \mathrm{O}_{8}$ nanoparticles were characterized by $\mathrm{X}$-ray diffraction, Fourier transform infrared spectroscopy, scanning electron microscopy and transmission electron microscopy. The optical properties were investigated by photoluminescence emission and Raman spectroscopy.
\end{abstract}

Key Words: Barium orthovanadate, Microwave-assited solvothermal synthesis, Nanoparticles, Luminescence, Raman spectroscopy. |

\section{INTRODUCTION}

Microwave synthesized materials have been recently attracted much interest, both theoretical and experimentally. Metal orthovanadates have been employed in microwave devices, photoluminescence, IR-laser, light-emitting diode, photocatalyst and ferroelectric devices ${ }^{1-3}$. The metal orthovanadates have an orthorhombic crystal structure. The magnetic ions $\mathrm{M}^{2+}$ form the so-called Kagome ladder, the $\mathrm{MO}_{6}$ octahedra are located in zigzag layers separated by nonmagnetic ions ${ }^{3} \mathrm{~V}^{5+}$. The manufacturing methods of metal orthovanadates have been developed to enhance the applications by a range of processes, such as a solid-state reaction ${ }^{4,5}$, a solution phase metathetic $\operatorname{method}^{6}$, sol-gel ${ }^{7}$, a solid-state metathesis approach ${ }^{8}$, a mechano-chemical method ${ }^{9}$ and a floating zone technique ${ }^{10}$. The concept of introducing chemical reactions between precursors powders has emerged as an attractive method to synthesize novel materials. Solid-state metathesis reactions require minimum energy input to initiate the synthesis reactions. There are several sources of providing the required energy such as contacting precursors with a heated filament, using electromagnetic waves. Microwave energy is delivered to the surface of the material by radiant and/or convection heating, which is transferred to the bulk of the material via conduction with an electromagnetic field ${ }^{11}$. Hydrothermal process is an efficient low temperature method that allows the formation of particles with high degree of crystallinity and easy dispersion in an aqueous medium. The use of microwave energy in hydrothermal system promotes the development of a rapid heating to the required temperature with rapid rates of crystallization ${ }^{12,13}$. Recently, microwave solvothermal processes ${ }^{14,15}$ have been reported the use of a facile and fast method in preparing nanocrystalline particles of metal tungstates with unique and enhanced properties.

Microwave-assisted solvothermal reactions provide a facile route for the synthesis of $\mathrm{Ba}_{3} \mathrm{~V}_{2} \mathrm{O}_{8}$ nanoparticles, which were obtained in the form of loosely connected nano-sized particles at considerably lower temperatures with a high pressure than those usually employed for their synthesis. Microwaveassisted solvothermal reaction involves the exchange of atomic/ ionic species, where the driving force is the exothermic reaction in ethylene glycol accompanying the formation of $\mathrm{NaCl}$ with a high lattice energy. The microwave exothermic reaction occurs so rapidly that the temperature and the pressure of the ethylene glycol increases so quickly that the reaction products are essentially heated up.

In this study, the $\mathrm{Ba}_{3} \mathrm{~V}_{2} \mathrm{O}_{8}$ nanoparticles were synthesized using a facile solvothermal route assisted by the microwave irradiation. The synthesized $\mathrm{Ba}_{3} \mathrm{~V}_{2} \mathrm{O}_{8}$ nanoparticles were characterized by X-ray diffraction, Fourier transform infrared spectroscopy, scanning electron microscopy and transmission electron microscopy. The optical properties were examined by photoluminescence emission and Raman spectroscopy.

\section{EXPERIMENTAL}

Fig. 1 shows a flow chart for the synthesis of $\mathrm{Ba}_{3} \mathrm{~V}_{2} \mathrm{O}_{8}$ nanoparticles by the microwave solvothermal process. $\mathrm{BaCl}_{2} \cdot 2 \mathrm{H}_{2} \mathrm{O}, \mathrm{Na}_{3} \mathrm{VO}_{4}$ and ethylene glycol of analytic reagent 
grade were used to prepare the $\mathrm{Ba}_{3} \mathrm{~V}_{2} \mathrm{O}_{8}$ compound. Each of $0.012 \mathrm{~mol} \mathrm{BaCl}{ }_{2} \cdot 2 \mathrm{H}_{2} \mathrm{O}$ and $0.008 \mathrm{~mol} \mathrm{Na}_{3} \mathrm{VO}_{4}$ for $\mathrm{Ba}_{3} \mathrm{~V}_{2} \mathrm{O}_{8}$ was dissolved in $30 \mathrm{~mL}$ ethylene glycol. The solutions were mixed and adjusted at a $\mathrm{pH} 9.5$ using $\mathrm{NaOH}$. The aqueous solution was stirred in ultrasonic bath at room temperature. In the sequence, the mixture was transferred into a Teflon lined digestion vessel of $120 \mathrm{~mL}$ capacity. The Teflon vessel was placed into a microwave solvothermal autoclave $(2.45 \mathrm{GHz}$, maximum power of $800 \mathrm{~W}$ ). The microwave solvothermal conditions were kept at $200{ }^{\circ} \mathrm{C}$ for $0.5 \mathrm{~h}$. After microwave solvothermal process, the microwave autoclave was cooled to room temperature. The resulting solutions were treated with ultrasonic radiation and washed many times with distilled hot water. The white precipitates were corrected and dried at $100{ }^{\circ} \mathrm{C}$ in a dry oven. The final products were heat-treated at $600{ }^{\circ} \mathrm{C}$ for $3 \mathrm{~h}$.

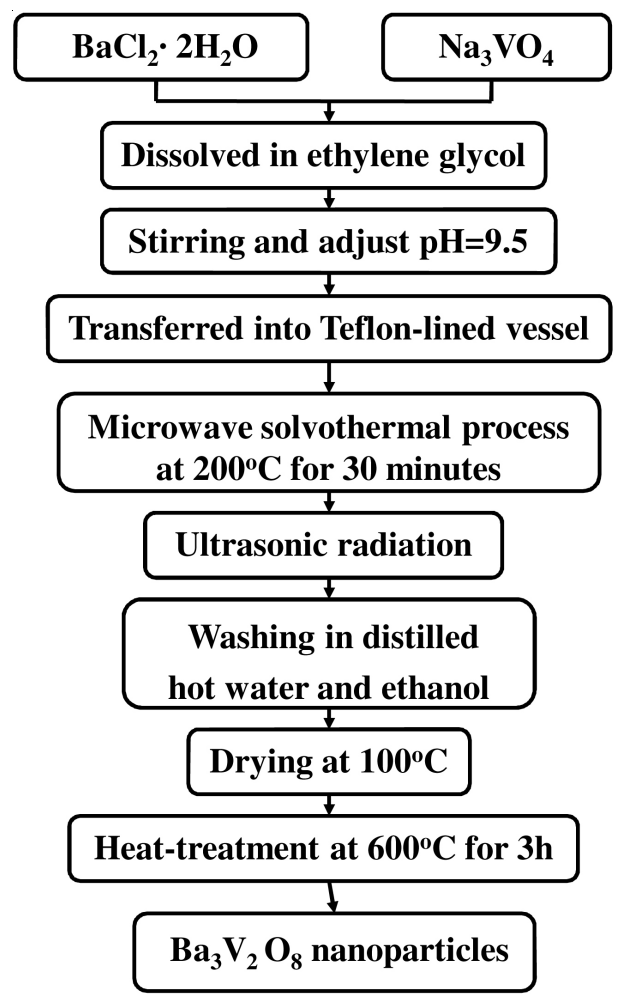

Fig. 1. Flow chart for the synthesis of $\mathrm{Ba}_{3} \mathrm{~V}_{2} \mathrm{O}_{8}$ nanoparticles by the microwave solvothermal process

The existing phases of the $\mathrm{Ba}_{3} \mathrm{~V}_{2} \mathrm{O}_{8}$ particles after the microwave solvothermal process were identified by powder XRD $\left(\mathrm{CuK}_{\alpha}\right.$, Rigaku D/MAX 2200, Japan). FTIR (Nicolet IR 200, Thermo Electron Corporation, USA) was used to examine the absorption behaviour of the synthesized $\mathrm{Ba}_{3} \mathrm{~V}_{2} \mathrm{O}_{8}$ particles over the frequency range, $4000-400 \mathrm{~cm}^{-1}$. The microstructure, particle morphology and qualitative compositions of the $\mathrm{Ba}_{3} \mathrm{~V}_{2} \mathrm{O}_{8}$ particles were observed by SEM (JSM-5600, JEOL, Japan) and TEM (JEM 2000-FX, $250 \mathrm{kV}$, Japan). The photoluminescence spectrum was recorded using a spectrophotometer (Perkin Elmer LS55, UK) at room temperature. Raman spectroscopy measurement was performed using LabRam HR (Jobin-Yvon, France). The $514.5 \mathrm{~nm}$ line of an Ar-ion laser was used as the excitation source, the power was kept at 0.5 $\mathrm{mW}$ on the samples.

\section{RESULTS AND DISCUSSION}

Fig. 2 shows XRD patterns of the $\mathrm{Ba}_{3} \mathrm{~V}_{2} \mathrm{O}_{8}$ nanoparticles synthesized by the microwave solvothermal process after heattreatment at $600{ }^{\circ} \mathrm{C}$ for $3 \mathrm{~h}$. All observed diffraction peaks could be assigned to the trigonal phases, which is in good agreement with the crystallographic data of $\mathrm{Ba}_{3} \mathrm{~V}_{2} \mathrm{O}_{8}$ (JCPDS: 71-2060, space group R-3m $)^{8}$. It suggests that microwave solvothermal synthesis is suitable for the growth of $\mathrm{Ba}_{3} \mathrm{~V}_{2} \mathrm{O}_{8}$ crystallites with the strongest major intensity peaks from the (015), (110) and (205) planes with some preferred orientation, respectively.

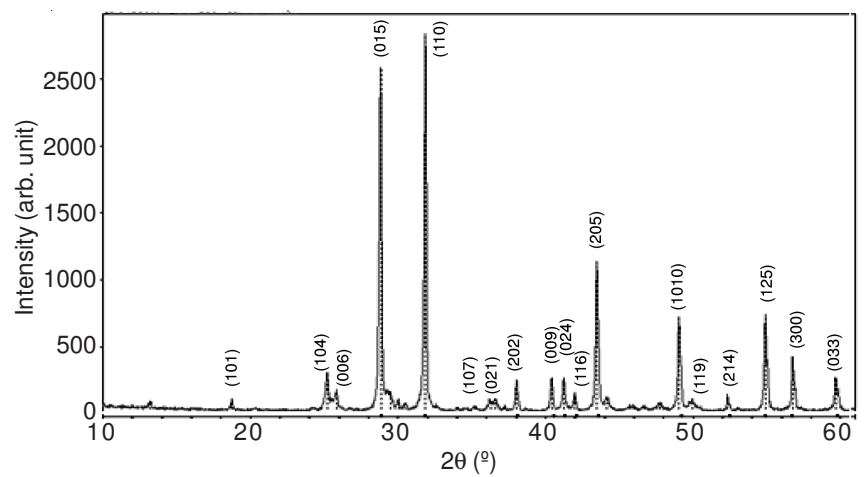

Fig. 2. XRD patterns of the $\mathrm{Ba}_{3} \mathrm{~V}_{2} \mathrm{O}_{8}$ nanoparticles

Fig. 3 shows FT-IR spectrum of the $\mathrm{Ba}_{3} \mathrm{~V}_{2} \mathrm{O}_{8}$ nanoparticles synthesized by the microwave solvothermal process in the wavenumber range, $4000-480 \mathrm{~cm}^{-1}$. The large isolated absorbable peak around $820 \mathrm{~cm}^{-1}$ reveals typical characteristics of a strong $\mathrm{V}-\mathrm{O}$ stretching in the $\left[\mathrm{VO}_{4}\right]^{3-}$. The strong V-O stretching peaks are contributed to the uniform regular $\left[\mathrm{VO}_{4}\right]^{3-}$ tetrahedron of the metal orthovanadates. The band at $1450 \mathrm{~cm}^{-1}$ is assumed that the samples prepared contain a small amount of surface-adsorbed water and alcohol.

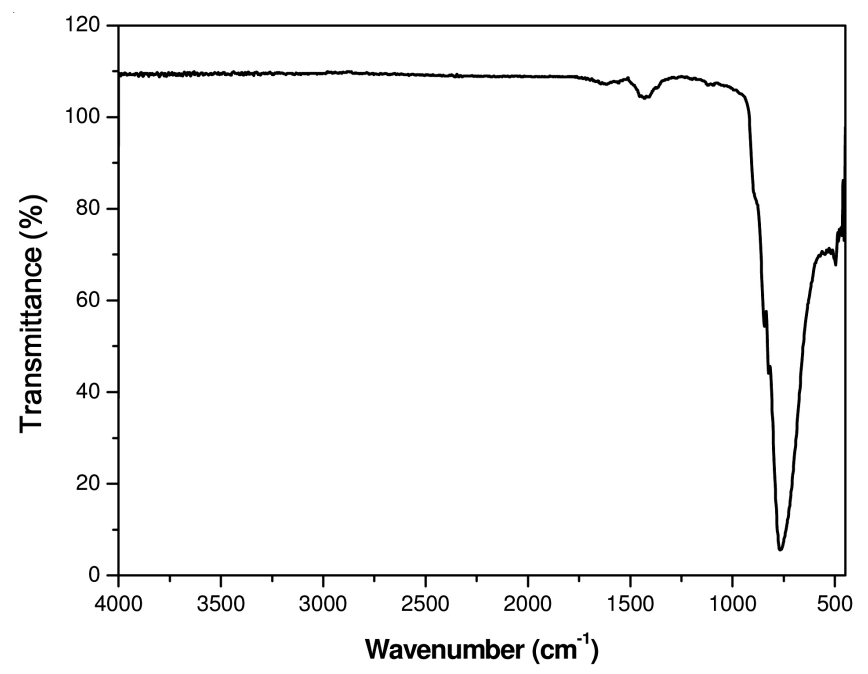

Fig. 3. FT-IR spectra of the $\mathrm{Ba}_{3} \mathrm{~V}_{2} \mathrm{O}_{8}$ nanoparticles

Fig. 4 shows a SEM image (a) and a TEM image (b) of the $\mathrm{Ba}_{3} \mathrm{~V}_{2} \mathrm{O}_{8}$ nanoparticles synthesized by the microwave solvothermal process. The SEM image of $\mathrm{Ba}_{3} \mathrm{~V}_{2} \mathrm{O}_{8}$ in Fig. 4(a) shows a well-defined and homogeneous morphology, while the TEM image of $\mathrm{Ba}_{3} \mathrm{~V}_{2} \mathrm{O}_{8}$ in Fig. 4(b) shows the particle 
sizes of $50-150 \mathrm{~nm}$. The solvothermal synthesis proceeds the reaction of $\mathrm{BaCl}_{2} \cdot 2 \mathrm{H}_{2} \mathrm{O}+\mathrm{Na}_{3} \mathrm{VO}_{4}$ in a hot ethylene glycol solution as a polar solvent with a boiling point of $197^{\circ} \mathrm{C}$. The microwave solvothermal process occurs in accordance with the reaction:

$$
3 \mathrm{BaCl}_{2} \cdot 2 \mathrm{H}_{2} \mathrm{O}+2 \mathrm{Na}_{3} \mathrm{VO}_{4} \rightarrow \mathrm{Ba}_{3} \mathrm{~V}_{2} \mathrm{O}_{8}+6 \mathrm{NaCl}+6 \mathrm{H}_{2} \mathrm{O}
$$

When the microwave radiation is supplied to the ethylene glycol under a sealed pressure above boiling point, the components dissolving in the ethylene glycol are charged and vibrated in electric field interdependently. The microwave solvothermal process is adjusted to heat the metal orthovanadates uniformly resulting in fine particles with a controlled morphology and to fabricate the product in a green manner without the generation of solvent waste. The microwaveassisted solvothermal reaction involves the exchange of atomic/ ionic species, where the driving force is the exothermic reaction in ethylene glycol accompanying the formation of $\mathrm{NaCl}$ with a high lattice energy. The microwave exothermic reaction occurs so rapidly that the temperature and the pressure of the ethylene glycol increases so quickly that the reaction products are essentially heated up. The microwave-assisted solvothermal reactions provide a facile route for the synthesis of $\mathrm{Ba}_{3} \mathrm{~V}_{2} \mathrm{O}_{8}$ nanoparticles, which were obtained in the form of loosely connected nano-sized particles at considerably lower temperatures with a high pressure than those usually employed for their synthesis. The well-defined $\mathrm{Ba}_{3} \mathrm{~V}_{2} \mathrm{O}_{8}$ nanoparticles features synthesized by the microwave-assisted solvothermal process have a control over the morphology of the fine particles and can be used for technological applications.
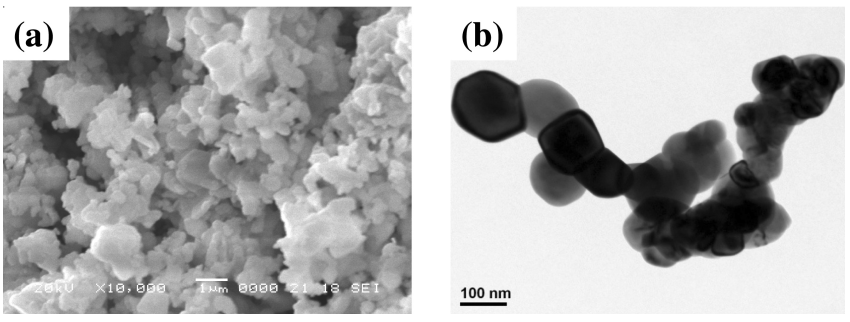

Fig. 4. A SEM image (a) and a TEM image (b) of the $\mathrm{Ba}_{3} \mathrm{~V}_{2} \mathrm{O}_{8}$ nanoparticles

Fig. 5 shows photoluminescence emission spectrum of the synthesized $\mathrm{Ba}_{3} \mathrm{~V}_{2} \mathrm{O}_{8}$ nanoparticles exited at $250 \mathrm{~nm}$ at room temperature. With excitation at $250 \mathrm{~nm}, \mathrm{Ba}_{3} \mathrm{~V}_{2} \mathrm{O}_{8}$ particles exhibit major photoluminescence emissions in the blue wavelength range of 390-430 $\mathrm{nm}$. The emissions of three narrow shoulders at $c a$. 490, 510 and $530 \mathrm{~nm}$ are considered to form by defect structures. The emission spectra of barium orthovanadates are due mainly to charge-transfer transitions within the $\left[\mathrm{VO}_{4}\right]^{3-}$ complex. The explanation of the narrow shoulders in Fig. 5 is proposed considering the Jahn-Teller splitting effect ${ }^{16,17}$ on excited states of $\left[\mathrm{VO}_{4}\right]^{3-}$ and anion in the $\mathrm{Ba}_{3} \mathrm{~V}_{2} \mathrm{O}_{8}$. This is similar to that reported by Zhan et al. ${ }^{18}$. The JahnTeller splitting effect essentially determines the emission shape of the $\mathrm{Ba}_{3} \mathrm{~V}_{2} \mathrm{O}_{8}$ particles. The additional emission bands can be interpreted by the existence of Frenkel defect structures (oxygen ion shifted to the inter-position with the simultaneous creation of vacancies) in the surface layers of the $\mathrm{Ba}_{3} \mathrm{~V}_{2} \mathrm{O}_{8}$ particles $^{19,20}$.

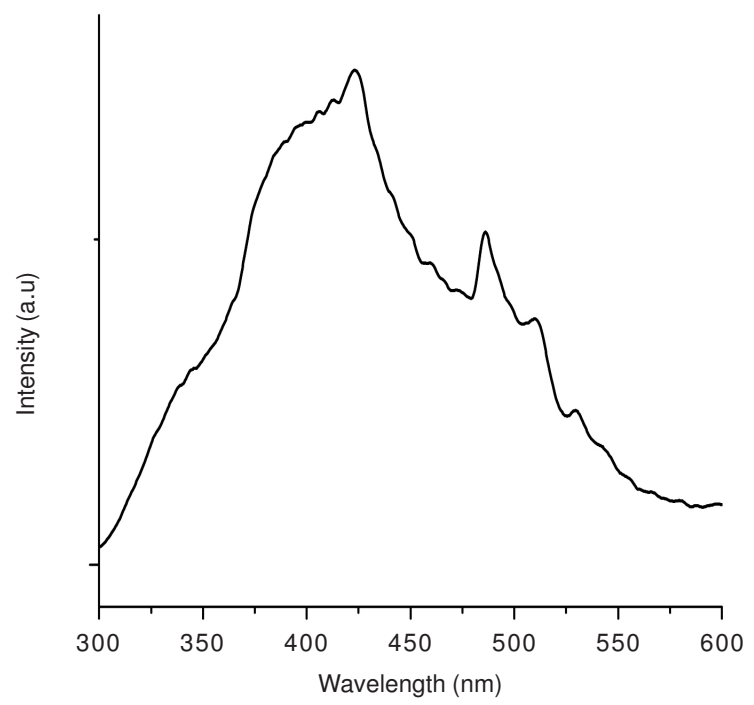

Fig. 5. Photoluminescence emission spectrumf the synthesized $\mathrm{Ba}_{3} \mathrm{~V}_{2} \mathrm{O}_{8}$ nanoparticles exited at $250 \mathrm{~nm}$ at room temperature

Fig. 6 shows Raman spectrum of the synthesized $\mathrm{Ba}_{3} \mathrm{~V}_{2} \mathrm{O}_{8}$ nanoparticles excited by the $514.5 \mathrm{~nm}$ line of an Ar-ion laser at $0.5 \mathrm{~mW}$ on the samples. The vibration modes in the Raman spectra of the $\mathrm{Ba}_{3} \mathrm{~V}_{2} \mathrm{O}_{8}$ nanoparticles are classified into two groups, internal and external. The internal vibrations are related to the $\left[\mathrm{VO}_{4}\right]^{3-}$ molecular group with a stationary mass center. The external vibrations or lattice phonons are associated to the motion of the $\mathrm{Ba}^{2+}$ and $\mathrm{Ca}^{2+}$ cation and rigid molecular units $^{21}$. The Raman modes for the $\mathrm{Ba}_{3} \mathrm{~V}_{2} \mathrm{O}_{8}$ particles were detected at $838,809,795,378,334$ and $334 \mathrm{~cm}^{-1}$, the free rotation mode was detected at $166 \mathrm{~cm}^{-1}$ and the external modes were localized at $123 \mathrm{~cm}^{-1}$. The well-resolved sharp peaks for the $\mathrm{Ba}_{3} \mathrm{~V}_{2} \mathrm{O}_{8}$ nanoparticles indicate that the synthesized particles are highly crystallized.

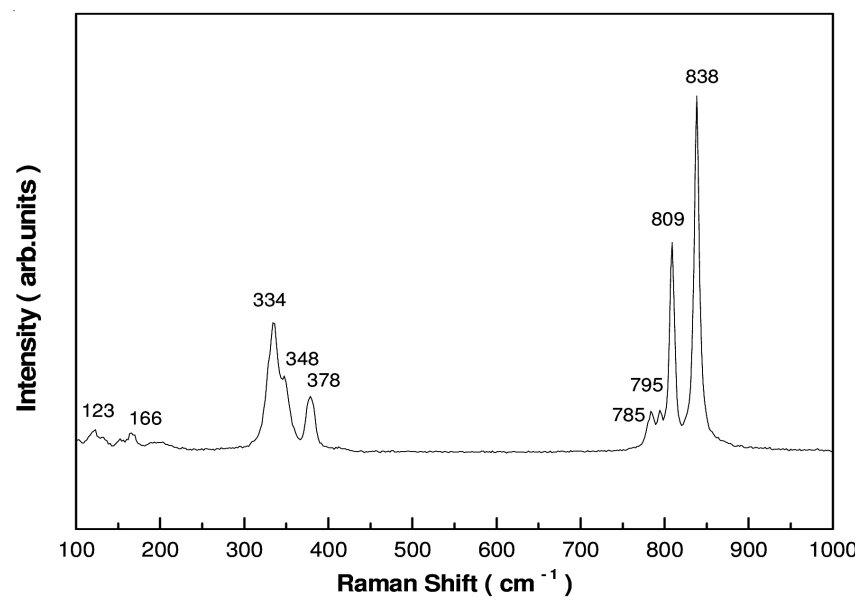

Fig. 6. Raman spectrum of the synthesized $\mathrm{Ba}_{3} \mathrm{~V}_{2} \mathrm{O}_{8}$ nanoparticles excited by the $514.5 \mathrm{~nm}$ line of an Ar-ion laser at $0.5 \mathrm{~mW}$ on the samples

\section{Conclusion}

Barium orthovanadate $\left(\mathrm{Ba}_{3} \mathrm{~V}_{2} \mathrm{O}_{8}\right)$ nanoparticles were synthesized successfully by the microwave solvothermal processes in a hot ethylene glycol solution as a polar solvent. The microwave solvothermal reactions occured so rapidly that the exothermic reaction was essentially used to heat up the 
metal orthovanadates. Well-crystallized $\mathrm{Ba}_{3} \mathrm{~V}_{2} \mathrm{O}_{8}$ nanoparticles were formed after heat-treatment at $600{ }^{\circ} \mathrm{C}$ for $3 \mathrm{~h}$ showing a fine and homogeneous morphology with particle sizes of 50$150 \mathrm{~nm}$. With excitation at $250 \mathrm{~nm}$, the $\mathrm{Ba}_{3} \mathrm{~V}_{2} \mathrm{O}_{8}$ nanoparticles exhibited major photoluminescence emissions in the blue wavelength range of 390-430 nm. The well-resolved Raman modes for the $\mathrm{Ba}_{3} \mathrm{~V}_{2} \mathrm{O}_{8}$ nanoparticles indicated that the synthesized particles were highly crystallized.

\section{ACKNOWLEDGEMENTS}

This study was supported by Basic Science Research Program through the National Research Foundation of Korea (NRF) funded by the Ministry of Education, Science and Technology (2012-0007858).

\section{REFERENCES}

1. T. Nakajima, M. Isobe, T. Tsuchiya, Y. Ueda and T. Kumagai, J. Luminescence, 129, 1598 (2009).

2. F. Yen, R.P. Chaudhury, E. Galstyan, B. Lorenz, Y.Q. Wang, Y.Y. Sun and C.W. Chu, Physica B, 403, 1487 (2008).

3. N. Rogado, G. Lawes, D.A. Huse, A.P. Ramirez and R.J. Cava, Solid State Commun., 124, 229 (2002).

4. D. Wang, Z. Zou, J. Ye and Res. Chem. Intermed., 31, 433 (2005)

5. M. Kurzawa and A. Blonska-Tabero, J. Therm. Anal. Calorim., 77, 17 (2004).
6. P. Parhi and V. Manivannan, Mater. Res. Bull., 43, 2966 (2008).

7. S.S. Kim, H. Ikuta and M. Wakihara, Solid State Ionics, 139, 57 (2001).

8. P. Parhi, V. Manivannan, S. Kohli and P. Mccurdy, Bull. Mater. Sci., 31, 885 (2008).

9. V. Manivannan, P. Parhi and J. Howard, J. Cryst. Growth, 310, 2793 (2008).

10. R. Szymczak, M. Baran, J. Fink-Finowicki, B. Krzymanska, P. Aleshkevych, H. Szymczak, S.N. Barilo, G.L. Bychkov and S.V. Shiryaev, J. Non-Cryst. Solids, 354, 4186 (2008).

11. S. Das, A.K. Mukhopadhyay, S. Datta and D. Basu, Bull. Mater. Sci., 32, 1 (2009).

12. K.P.F. Siqueira, R.L. Moreira, M. Valadares and A. Dias, J. Mater. Sci., 45, 6083 (2010).

13. J.C. Sczancoski, L.S. Cavalcante, M.R. Joya, J.A. Varela, P.S. Pizani and E. Longo, Chem. Eng. J., 140, 632 (2008).

14. J. Bi, L. Wu, Z. Li, Z. Ding, X. Wang and X. Fu, J. Alloys Comp., 480, 684 (2009).

15. T. Thongtem, A. Phuruangrant and S. Thongtom, Curr. Appl. Phys., 8, 189 (2008)

16. Y. Toyozawa and M. Inoue, J. Phys. Soc. Jpn., 21, 1663 (1966).

17. E.G. Reut, Izv. Akad. Nauk SSSR, Ser. Fiz., 43, 1186 (1979).

18. Y. Zhang, N.A.W. Holzwarth and R.T. Williams, Phys. Rev. B, 57, 12738 (1998).

19. J. Van Tol and J.H. Van Der Waals, Mol. Phys., 88, 803 (1996).

20. V.B. Mikhailik, H. Kraus, D. Wahl and M.S. Mykhaylyk, Phys. Status Solid B, 242, R17 (2005).

21. T.T. Basiev, A.A. Sobol, P.G. Zverev, L.I. Ivleva, V.V. Osiko and R.C. Powell, Opt. Mater., 11, 307 (1999). 